
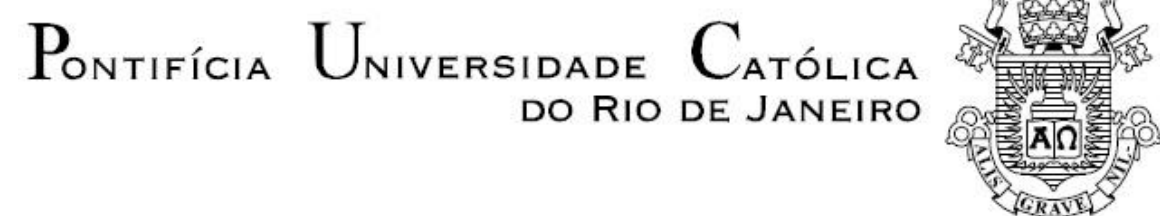

Íris Firmino Cardoso

\title{
Avaliação Preliminar dos Movimentos Aéreos no Aeroporto Internacional Antônio Carlos Jobim - Galeão
}

Dissertação de Mestrado

Dissertação apresentada como requisito parcial para obtenção do grau de mestre pelo Programa de Pós-graduação em Engenharia de Produção do Departamento de Engenharia Industrial da PUC-Rio.

Orientadora: Profa. Fernanda Maria Pereira Raupp

Rio de Janeiro

Novembro de 2010 


\section{Íris Firmino Cardoso}

\section{Avaliação Preliminar dos Movimentos Aéreos no Aeroporto Internacional Antônio Carlos Jobim - Galeão}

Dissertação apresentada como requisito parcial para obtenção do grau de mestre pelo Programa de Pós-graduação em Engenharia de Produção do Departamento de Engenharia Industrial da PUC-Rio. Aprovada pela Comissão Examinadora abaixo assinada.

Profa. Fernanda Maria Pereira Raupp

Orientadora

Departamento de Engenharia Industrial - PUC-Rio

Profa. Flavia Cesar Teixeira Mendes Departamento de Engenharia Industrial - PUC-Rio

Prof. Nelio Domingues Pizzolato

Diretor do Departamento de Engenharia Industrial - PUC-Rio

Prof. José Eugenio Leal

Coordenador Setorial do Centro Técnico Científico - PUC-Rio

Rio de Janeiro, 18 de novembro de 2010 
Todos os direitos reservados. É proibida a reprodução total ou parcial do trabalho sem autorização da universidade, do autor e do orientador.

\section{Íris Firmino Cardoso}

Bacharel em Ciências Estatísticas na Escola Nacional de Ciências Estatísticas (ENCE).

Ficha Catalográfica

Cardoso, Íris Firmino

Avaliação preliminar dos movimentos aéreos no Aeroporto Internacional Antônio Carlos Jobim Galeão / Íris Firmino Cardoso ; orientador: Fernanda Maria Pereira Raupp. - 2010.

94 f. : il. (color.) ; $30 \mathrm{~cm}$

Dissertação (mestrado)-Pontifícia Universidade Católica do Rio de Janeiro, Departamento de Engenharia Industrial, 2010.

Inclui bibliografia

1. Engenharia Industrial - Teses. 2. Aeroporto. 3. Simulação. 4. Movimento aéreo. 5. ProModel. I. Raupp, Fernanda Maria Pereira. II. Pontifícia Universidade Católica do Rio de Janeiro. Departamento de Engenharia Industrial. III. Título. 
À minha mãe e ao futuro que me aguarda. 


\section{Agradecimentos}

À minha mãe, pelo constante apoio e incentivo.

Aos amigos, por compreenderem e aceitarem meus momentos de ausência.

Aos colegas de curso, pela ajuda nas horas de estudo e pelas gargalhadas sempre que se faziam necessárias.

Aos professores e funcionários do departamento, por tornarem possível a conclusão dessa etapa da minha vida.

Ao programa de bolsas de estudo da CAPES.

À todos, que de forma direta ou indireta, contribuíram para mais essa conquista. 


\section{Resumo}

Cardoso, Íris Firmino; Raupp, Fernanda Maria Pereira. Avaliação Preliminar dos Movimentos Aéreos no Aeroporto Internacional Antônio Carlos Jobim - Galeão. Rio de Janeiro, 2010. 94p. Dissertação de Mestrado - Departamento de Engenharia Industrial, Pontifícia Universidade Católica do Rio de Janeiro.

A técnica de simulação tem sido largamente utilizada como uma ferramenta no processo de tomada de decisão. O presente trabalho propõe o uso dessa ferramenta para avaliar o desempenho atual do Aeroporto Internacional do Rio de Janeiro Antônio Carlos Jobim (Galeão) no que se refere à movimentação de chegadas e de saídas de aeronaves, estimando, entre outras medidas, o ponto de saturação do Aeroporto, e conseqüentemente avaliar o nível de serviço prestado atualmente, visando, caso necessário, propor alterações em sua infraestrutura. $\mathrm{O}$ software utilizado na simulação é apresentado e explicado detalhadamente a fim de descrever a seqüência de procedimentos adotados para a obtenção dos resultados desse estudo. Para estimação dos parâmetros necessários na simulação, serão utilizados dados atuais, em forma de séries de tempo, referentes aos processos de movimentação de aeronaves no Aeroporto do Galeão, bem como dados sobre infraestrutura disponível no local.

\section{Palavras-chave}

Aeroporto; Simulação; Movimento Aéreo; ProModel 


\section{Abstract}

Cardoso, Íris Firmino; Raupp, Fernanda Maria Pereira (Advisor). Preliminary Evaluation of Air Movements at the International Airport Antonio Carlos Jobim - Galeão. Rio de Janeiro, 2010. 94p. MSc Dissertation - Departamento de Engenharia Industrial, Pontifícia Universidade Católica do Rio de Janeiro.

The simulation technique has been widely used as a tool in the decision making process. The present work proposes the use of this tool to evaluate the current performance of the International Airport of Rio de Janeiro Antonio Carlos Jobim (Galeão) concerning the aircraft movements of arrivals and departures, estimating, among other measures, the saturation point of the airport, and consequently evaluating the current level of service provided, aiming, if necessary, to propouse modifications in its infrastructure. The software used in the simulation is presented and explained in details in order to describe the sequence of procedures adopted to obtain the results of this study. For the estimation of the necessary parameters in the simulation, recent data, in the form of time series, of the aircraft movement procedures in the Galeão Airport, will be used as well as local infrastructure data locally available.

\section{Keywords}

Airport; Simulation; Air Movement; ProModel 


\section{Sumário}

1 Introdução 13

2 Sistema Aeroportuário Brasileiro 15

2.1 A INFRAERO 16

2.2 Aeroporto Internacional Antônio Carlos Jobim (Galeão) 18

2.2.1 Terminal de passageiros 20

2.2.2 Terminal de carga 22

3 Revisão Bibliográfica $\quad 24$

$4 \quad$ Metodologia de Pesquisa 26

$\begin{array}{lll}4.1 & \text { Teoria das Filas } & 27\end{array}$

4.2 Simulação 28

4.2.1 Tipos de Simulação 30

4.2.2 Linguagens de Simulação 30

4.3 Análise Estatística em Simulação 40

5 O Desempenho Atual 42

5.1 Análise Exploratória dos Dados 42

5.2 Avaliação do Nível de Serviço Prestado no Período 46

5.3 Validação da Simulação $\quad 54$

6 Aplicação do modelo: Galeão 58

6.1 Modelagem do Sistema 58

6.2 Resultados 68

7 Conclusões $\quad 76$

$8 \quad$ Referências Bibliográficas $\quad 78$

9 Glossário $\quad 81$

Apêndice $\quad 82$ 


\section{Lista de Figuras}

Figura 1: Aeroporto Internacional Antônio Carlos Jobim (fonte:

INFRAERO)

Figura 2: Tela inicial do ProModel

Figura 3: Opções do Software

Figura 4: Menu Construir

Figura 5: Definindo os Locais 35

Figura 6: Definindo as Entidades 36

Figura 7: Definindo as Chegadas das Entidades 37

Figura 8: Definindo os Processos 38

Figura 9: Configurando a Simulação 39

Figura 10: INFRAERO - Voos Online 43

Figura 11: Fluxograma representativo do Sistema - Aterrissagens 62

Figura 12: Fluxograma representativo do Sistema - Decolagens 63

Figura 13: Fluxograma representativo do Sistema - Estacionamento $\quad 65$

$\begin{array}{ll}\text { Figura 14: Fluxograma representativo do modelo } & 67\end{array}$ 


\section{Lista de Gráficos}

Gráfico 1: 28 de Janeiro de $2010 \quad 46$

Gráfico 2: 29 de Janeiro de $2010 \quad 47$

Gráfico 3: 30 de Janeiro de 2010

Gráfico 4: 31 de Janeiro de $2010 \quad 48$

Gráfico 5: 1 de Fevereiro de 2010

Gráfico 6: 2 de Fevereiro de $2010 \quad 49$

Gráfico 7: 3 de Fevereiro de $2010 \quad 49$

Gráfico 8: 4 de Fevereiro de 2010

Gráfico 9: 5 de Fevereiro de $2010 \quad 50$

Gráfico 10: 6 de Fevereiro de 2010

Gráfico 11: 7 de Fevereiro de 2010

Gráfico 12: 8 de Fevereiro de 2010

Gráfico 13: 9 de Fevereiro de 2010

Gráfico 15: Simulação Dia $1 \quad 68$

Gráfico 16: Simulação Dia $2 \quad 69$

Gráfico 17: Simulação Dia $3 \quad 69$

Gráfico 18: Simulação Dia 4

Gráfico 19: Simulação Dia 5

Gráfico 20: Simulação Dia 6

Gráfico 21: Simulação Dia 7

Gráfico 22: Simulação Dia 8

Gráfico 23: Simulação Dia 9

Gráfico 24: Simulação Dia 10

Gráfico 25: Simulação Dia 11

Gráfico 26: Simulação Dia 12 


\section{Lista de Quadros}

Quadro 1: Classificação do fluxo aéreo segundo o atraso médio das aeronaves 42

Quadro 2: Situação observada no Galeão 53

Quadro 3: Resultados da análise de Regressão Linear 56

Quadro 4: Divisão da Base de Dados 59

Quadro 5: Estimativas para Chegada/Partida de Aeronaves 61

Quadro 6: Situação obtida na simulação 74 


\section{Lista de Tabelas}

Tabela 1: Aeródromos em território nacional por estado segundo $\begin{array}{ll}\text { tipo e administração } & 15\end{array}$

Tabela 2: Dados relativos aos Terminais de Passageiros do Galeão 21

Tabela 3: Dados segundo o Status do Voo 44

Tabela 4: Estatísticas do horário de Confirmação dos Voos 45

Tabela 5: Voos segundo Terminal e Tipo 55 